\title{
Beyond Interpretation: The Unmet Need for Linguistically and Culturally Competent Care for Latinx People Living with HIV in a Southern Region with a Low Density of Spanish Speakers
}

\author{
Jacqueline E. Sherbuk, ${ }^{1}$ Kristen Petros de Guex, ${ }^{1}$ Diego Anazco Villarreal, ${ }^{2}$ Sarah Knight, ${ }^{1}$ \\ Kathleen A. McManus, ${ }^{1}$ Tabor Flickinger, ${ }^{3}$ and Rebecca Dillingham ${ }^{1}$
}

\begin{abstract}
Latinx people living with HIV (PLWH) experience disparities in health outcomes and face unique barriers to care related to language, intersectional stigma, and immigration status. We aimed to explore the lived experience of Spanish-speaking Latinx PLWH in the nonurban South to better understand how to improve care for this minority language population. We conducted semistructured interviews with 22 participants (10 men, 10 women, 2 transgender women) who were recruited from a Ryan White HIV/AIDS program (RWHAP) and a community-based organization. Almost all participants were foreign born. Emerging themes included language barriers, cultural differences, inadequate interpreter services, HIV-related and intersectional stigma, isolation, and relationships as a source of support. These barriers may contribute to disparities in outcomes for Latinx PLWH. New interventions are needed to overcome barriers, foster community, and ensure culturally tailored models of care. Potential clinic-level interventions include the development of specialized training for RWHAP interpreters and inclusion of interpreters in care teams.
\end{abstract}

Keywords: HIV, Latinx, limited English proficient, barriers to care, interpreter services

\section{Introduction}

$\mathbf{L}$ ATINXS ARE DISPROPORTIONATELY affected by HIV, accounting for $27 \%$ of new HIV diagnoses in the United States in 2018 , but only $18 \%$ of the population. ${ }^{1,2}$ Latinx people living with HIV (PLWH) are diagnosed at a more advanced stage of disease $\mathrm{s}^{3,4}$ and have lower rates of retention in care ${ }^{5}$ than non-Hispanic white PLWH. Currently, only half of Latinx PLWH are virally suppressed, ${ }^{6}$ far below national goals for viral suppression. ${ }^{7}$ Improving and optimizing HIV care for Latinx PLWH is essential if the United States wants to end the HIV epidemic.

In the United States, Latinx PLWH are a diverse population representing varied native countries, lifestyles, and cultural norms, but, as a group, they face unique barriers to care. $^{8,9}$ Latinx PLWH encounter intersectional stigma related to HIV, ethnicity, gender, sexual orientation, and immigration. ${ }^{10}$ Language barriers for those who are limited English proficient (LEP) impact access to care, quality of care, satisfaction with care, and patient safety. ${ }^{11}$ Structural racism affects individual-level health decisions and behaviors among Latinx PLWH. ${ }^{12}$ Foreign-born Latinx PLWH have additional immigration-related barriers, including fear of deportation, lack of health insurance coverage, poverty, mistrust of support services, and limited support systems. $^{13-15}$

The HIV epidemic continues in the United States and over half of new HIV cases are occurring in the South, ${ }^{16}$ with a disproportionate increase in diagnoses among Latinx men. ${ }^{16,17}$ A higher proportion of cases in the South are nonurban, ${ }^{18}$ and Latinx PLWH in rural areas of the South may experience higher mortality than urban counterparts. ${ }^{19}$ As the HIV epidemic shifts to nonurban regions, a better understanding of the rural HIV epidemic is needed. ${ }^{20}$

\footnotetext{
${ }^{1}$ Division of Infectious Diseases and International Health, University of Virginia, Charlottesville, Virginia, USA.

${ }^{2}$ Colegio de Ciencias de la Salud, Escuela de Medicina, Universidad San Francisco de Quito, Quito, Ecuador.

${ }^{3}$ Division of General, Geriatric, Palliative, and Hospital Medicine, University of Virginia, Charlottesville, Virginia, USA.
} 
Our Ryan White HIV/AIDS program (RWHAP) clinic in the South serves a predominately nonurban population with a low density of LEP Spanish speakers. Our objective was to explore the lived experience of Latinx PLWH in our region. Using a qualitative approach, we aimed to understand how linguistic and cultural differences may impact the care of Latinx PLWH.

\section{Methods}

\section{Study design and population}

This study was approved by the University of Virginia Institutional Review Board for Health Sciences Research. The study population was Spanish-speaking Latinx PLWH ages 18 and older. Participants could speak English in addition to Spanish. Participants were recruited over the telephone and in person from two sites, (1) a RWHAP clinic located at a tertiary care hospital in the nonurban South and (2) a Latinx-serving community health organization. Convenience sampling was used. Informed consent was obtained from all participants.

\section{Data collection}

Semistructured interviews were performed in the clinic by two bilingual Spanish-speaking study personnel, one male and one female. Interview questions included basic demographic information, including age; place of birth; education level; time in the United States; language spoken at home, at work, and with friends; as well as time since HIV diagnosis.

Participants were then asked a series of open-ended questions regarding their experience living with HIV using an interview guide. The interview guide was developed based on health disparity frameworks for HIV care, which emphasize the interconnection of individual-level factors and social-structural factors on outcomes ${ }^{21,22}$ These factors include socioeconomic position, stigma, discrimination, stress, and social capital, all of which impact health behaviors and access to care, especially for vulnerable populations. The guide included items related to barriers that PLWH face in engagement in care and treatment, the role of social support in HIV care, and health care disparities for Latinx PLWH (Supplementary Table S1). Interviews ranged in length from 21 to $65 \mathrm{~min}$.

Interviews were audiotaped, transcribed, and translated. Two members of the study team, one a native Spanish speaker and the other a native English speaker with national medical interpretation certification, performed the translations. All translated interviews were reviewed by a second Spanish-speaking team member, and when concerns arose regarding the translation, team members returned to the audio transcript for clarification.

\section{Data analysis}

Translated interviews were imported into Dedoose for analysis [Dedoose Version 8.0.35 (2018); SocioCultural Research Consultants, LLC, Los Angeles, CA, www.dedoose .com]. An initial codebook was developed using grounded theory. Each interview was coded by at least two members of the study team. Codes and descriptions applied inconsistently by study team members were revised, and additional codes added as needed. The codebook was refined until good in- terrater reliability was achieved with a kappa statistic of 0.74 . Data saturation occurred after analysis of 18 interviews. The final codebook was applied to all interviews. (Supplementary Table S2)

\section{Results \\ Participant characteristics}

Twenty-eight individuals were invited to participate and $22(79 \%)$ enrolled in the study. Reasons given for nonparticipation included a focus on honing English language skills $(n=1)$, a general lack of interest in the study $(n=2)$, work conflicts $(n=1)$, transportation burden $(n=1)$, and confidentiality concerns $(n=1)$.

The 22 participants included 10 men, 10 women, and 2 transgender women (Table 1). The average age was 41.1 years [standard deviation (SD) 11.6 years]. All participants were born outside the continental United States, with the most common countries of origin being Honduras $(n=7)$, Mexico $(n=4)$, and El Salvador $(n=4)$. Participants had lived in the United States for a mean of 12.2 years (SD 9.8 years) with a range of 0.5 to 45 years. Fourteen participants (64\%) reported limited or no English proficiency, and 20 (91\%) spoke only Spanish at home. Participants reported a mean of 9.2 years since HIV diagnosis, with a range of 5 months to 35 years.

\section{Emerging themes from qualitative analysis}

Using grounded theory for analysis, emerging themes included language, cultural differences, inadequate interpreter services, stigma, isolation, and immigration (Table 2).

Language. Language was the most commonly identified theme, impacting most aspects of participants' health care, including access to care, communication with providers and support staff, and connection to other PLWH. Participants described challenges accessing care, including clinic phone recordings in English only and lack of Spanish-speaking scheduling staff.

Patient/provider language discordance impacted the quality of communication with providers. The inability to fully explain medical needs to providers resulted in hesitancy to raise concerns or confide in providers. "I can't be myself with the doctor," one participant admitted, "for the simple reason that she doesn't speak Spanish well, [and] I don't speak English well...She's not going to understand me. I'm better off not telling her anything."

Even when providers had intermediate Spanish proficiency, barriers arose during communication. One participant described an interaction with their doctor in the following way: "She takes out the translator on the computer and she translates a word that she doesn't understand and, then, done. But it's more time-consuming, because I know-I have the idea that there are more patients after me, so she has to go. So, that's why I tell her, 'No, well, I'm fine.' ... I can't express to her everything that I'm feeling. Not really-feeling, I mean in terms of my health."

Due to the frustration of being unable to communicate with health care providers, participants expressed a desire to learn English. One participant pursued English courses to advocate for himself: "If we are [expecting] for everyone to translate 
Table 1. Participant Characteristics

\begin{tabular}{|c|c|}
\hline Characteristic & $\mathrm{n}$ \\
\hline \multicolumn{2}{|l|}{ Gender } \\
\hline Male & 10 \\
\hline Female & 10 \\
\hline Transgender & 2 \\
\hline \multicolumn{2}{|l|}{ Age } \\
\hline Mean (SD), years & $41.1(11.6)$ \\
\hline \multicolumn{2}{|l|}{ Age group } \\
\hline $18-29$ years & 3 \\
\hline $30-39$ years & 7 \\
\hline $40-49$ years & 6 \\
\hline $50-59$ years & 4 \\
\hline $60+$ years & 2 \\
\hline \multicolumn{2}{|l|}{ Country of origin } \\
\hline Honduras & 7 \\
\hline Mexico & 4 \\
\hline El Salvador & 4 \\
\hline Guatemala & 2 \\
\hline Argentina & 1 \\
\hline Dominican Republic & 1 \\
\hline Peru & 1 \\
\hline United States (Puerto Rico) & 1 \\
\hline Venezuela & 1 \\
\hline \multicolumn{2}{|l|}{ Age on migration to United States } \\
\hline Mean (SD), years & $28.8(9.1)$ \\
\hline Range, years & 13 to 50 \\
\hline \multicolumn{2}{|l|}{ Time living in the United States } \\
\hline Mean (SD), years & $12.2(9.8)$ \\
\hline Range, years & 0.5 to 45 \\
\hline \multicolumn{2}{|l|}{ Time since HIV diagnosis } \\
\hline Mean (SD), years & $9.2(9.1)$ \\
\hline Range, years & 0.4 to 35 \\
\hline \multicolumn{2}{|l|}{ English proficiency } \\
\hline None & 9 \\
\hline Limited & 5 \\
\hline Basic & 4 \\
\hline Advanced & 4 \\
\hline \multicolumn{2}{|l|}{ Language spoken at home } \\
\hline Spanish only & 20 \\
\hline English and Spanish & 0 \\
\hline English only & 2 \\
\hline \multicolumn{2}{|l|}{ Language spoken with friends } \\
\hline Spanish only & 15 \\
\hline English and Spanish & 5 \\
\hline English only & 0 \\
\hline Other & 2 \\
\hline \multicolumn{2}{|l|}{ Language spoken at work } \\
\hline Spanish only & 6 \\
\hline English and Spanish & 9 \\
\hline English only & 3 \\
\hline Not currently working & 4 \\
\hline \multicolumn{2}{|l|}{ Education level } \\
\hline Primary school (fifth grade) & 5 \\
\hline Middle school (sixth-eighth) & 4 \\
\hline Some high school & 3 \\
\hline High school graduate/GED & 8 \\
\hline College & 2 \\
\hline
\end{tabular}

GED, general education diploma; SD, standard deviation. all the time, at the end of the day we'll never get the truth...That's why I went to school three years ago. I can't speak English perfectly, but I can get by.",

Cultural differences. While language presents a formidable barrier to optimal patient/provider communication, cultural differences in the expression of health concerns may also impede communication, and communication may lack the warmth desired by patients.

For one patient whose provider speaks Spanish, "Even though [my doctor] speaks Spanish, she doesn't understand a lot of things the way I am expressing them to her. So, that's a limitation because it doesn't allow for good communication or fluid communication with her...the contact I have with the doctor or with the providers isn't as charismatic as one is used to [in my home country]." Another participant described modifying her behavior to meet typical cultural expectations in the United States: "There's also a communication barrier because we [Latinos] don't know how to communicate. We usually go around in circles before getting to the point. And thanks to this culture, we've been taught to cut to the chase."

Interpretation services. Interpretation services were frequently used. For some participants, readily available professional interpreter services were sufficient to meet their needs and overcome the language barrier. However, for many, use of interpreter services brought about additional challenges. A common concern regarded the faithfulness of the interpreted message to the original. One participant noted that, "when you don't speak in English, it can be difficult because you can get frustrated when the message is not translated as expected, and if the interpreter can't use the words, it loses some strength and information."

The presence of the interpreter during a private medical visit led participants to question the confidentiality of the visit and hesitate to confide personal information to their providers. One participant described a time when he did not fully disclose his recent sexual activity, in part due to misinterpretation: "The doctor just asks me, 'Do you have a new partner?' My answer is no. But...maybe she's not asking the right questions? Maybe she isn't going deep enough to get at the information in a way that inspires trust? I mean, whether or not my sexual life is active, she doesn't know. She just asks, 'Do you have a partner or not?' Well, no. But... I mean, I'm sexually active...I'm having sex." The word "partner," often used in the phrase "sexual partners" to inquire about anyone with whom the patient has engaged in sexual activity, was interpreted as "pareja," which in Spanish connotes a long-term relationship. The patient, while possibly aware of this incomplete translation, chose to answer the question as it was literally posed, leading to miscommunication of medically relevant information.

While most participants reported helpful and professional interactions with medical interpreters, two participants had experienced negative, stigmatized interactions with interpreters, including one participant who recalled: "The [clinic interpreter] who told me [about my HIV diagnosis], she said to me, 'Listen, girl! You're going to die.' And it was very hard. I think they should have told me some other way." Another participant, diagnosed earlier in the AIDS epidemic, remembered: "The interpreter would wear an astronaut suit just to be in the same room as us, not even touching us. Even 


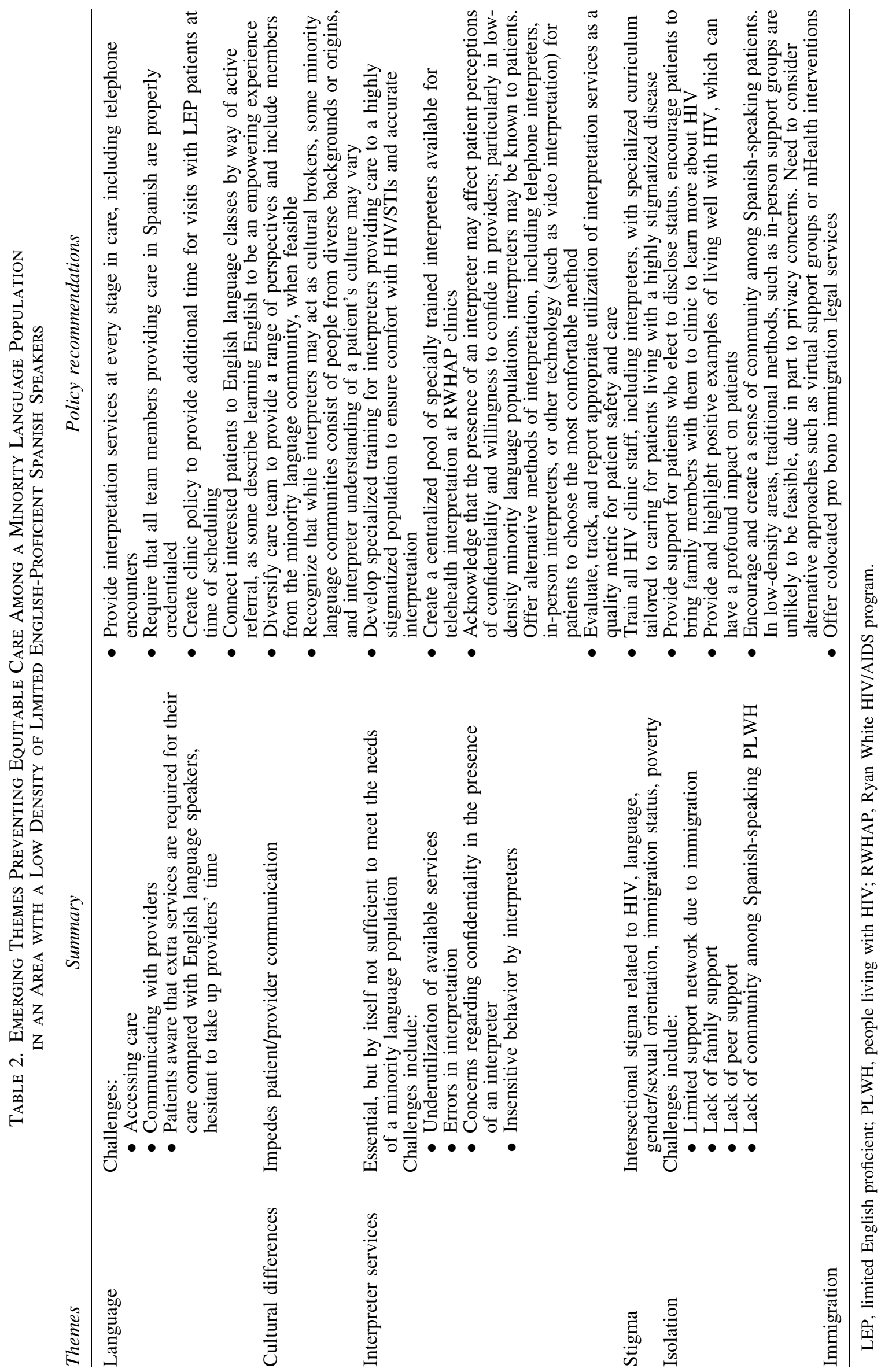


the social worker thought that was shocking, but he told me: 'This is what we have,' and those were the requests of the translator."

HIV-related and intersectional stigma. Internalized, anticipated, and experienced stigma affected participant experiences of living with HIV, from the initial diagnosis to their current care. For most participants, the initial reaction to receiving a diagnosis of HIV included feelings of uncertainty, shock, isolation, and a pervasive sense of imminent death. One participant explained, "The first thing you think is, 'I'm going to die.' It's what you hear everywhere. HIV/AIDS is associated with death." For those diagnosed early in the AIDS epidemic, feelings of imminent death were reinforced by health care providers. One participant recalled: "The doctor who gave me my results told me in a cold way that it was AIDS and that it was a disease that had no cure, and since there was practically no treatment, that I had more or less three months left to live."

Denial of the diagnosis was a common response. "I've never wanted to accept that it exists inside of me," admitted one participant, while another recounted, "I call it, "my inner enemy.", After diagnosis, many participants reported mental health concerns associated with the stigma of HIV, including depression, anxiety, and suicidal ideation. One participant recalled, "I want to die and people will say he committed suicide or something, instead of saying he died of HIV. Instead of saying he died of AIDS.",

Stigma led participants to be cautious about how they seek care and to avoid bringing home resources or medication bottles that may result in inadvertent disclosure. One participant described each clinic visit as a potential opportunity for disclosure, noting that "more than anything, when I came here for the first time to see the doctor, I was afraid that there would be people here that knew me and that they'd ask me, because I know that there are Hispanic people here-there are people close to me who work here, who work in cleaning services, cleaning up. So, every time that I come, I'm careful about that, so that they don't see me."

Anticipated stigma prevented many participants from disclosing their status to family and friends. Others felt that disclosing their HIV status would place a burden on family members due to the associated stigma and expressed concerns for their family members' reactions. Despite advances in knowledge regarding HIV transmission, people are "still living in the old days, when someone says HIV_- 'Don't hang out with him!' or 'Don't drink from the same glass,' or 'Don't touch him,' or 'Don't kiss him because he's going to infect you.' People still have that mentality...there's a lot of ignorance.',

In addition to stigma related to HIV, participants also faced intersectional stigma related to limited English proficiency, gender or sexual orientation, and immigration status. For one participant, "When you are a transgender woman, like myself, it's hard for doctors to understand you. It is more degrading than [when they are] treating a gay person; there is more rejection. They don't care about you. And you feel really depressed about that." One participant was able to access care, but perceived being a Spanish speaker to be a source of stigma in the medical setting. She explained that there are "people who don't like it when we speak Spanish. It bothers them... And you feel like you don't have a right to say anything because you're receiving assistance, and this is the only thing that should matter-no matter how they treat you. But this is false-it's the language barrier."

Isolation. Feelings of isolation were common and attributed to limited disclosure of participants' HIV diagnosis and a limited support network. Most participants described a limited number of family members to whom they had disclosed their status. One participant felt he was "drifting apart" from family "due to the problem I am facing" and the stress of keeping his diagnosis a secret. Support groups for PLWH were found to be inaccessible due to limited English proficiency. One participant noted that "not many people who speak Spanish talk about their illness, not that I know of anyway, so, no, I don't believe I have a support network."

Immigration. The impact of immigration on HIV care differed based on time since immigration and immigration status. Recent immigrants noted inability to work or access resources. One described the challenges faced as an undocumented immigrant after HIV diagnosis: "Here, you are illegal. You don't have documents, you don't have insurance. Here, you just have God and nothing else." Two participants, who immigrated over a decade ago, were aware of prior regulations prohibiting people with HIV from entering the United States. Other participants who were engaged in HIV care in their home countries lost their support systems upon immigrating, leading to depression, anxiety, and lack of motivation to remain adherent with treatment.

Sources of support and motivation. Relationships were identified as participants' primary source of support and motivation. Positive relationships included people who were aware of the HIV diagnosis and able to provide support as family members, friends, care team members, or other Spanish-speaking PLWH. Participants who were able to share their diagnosis with family and friends found considerable support in these relationships. One participant whose family accepted his diagnosis shared: "The most important thing has always been my family... That's what has sustained me up to this moment with the motivation to keep living."

Counseling provided at the time of diagnosis can aid in acceptance of the diagnosis, be a source of comfort, and provide a way to "get a little bit of hope back," as one participant described. She continued, "they explained to me that HIV wasn't like it used to be before, because there didn't used to be very many medications. So, they said, 'If you take your medications as directed, you're going to be fine'... And I took the advice and that's what I've done for all these years." Ongoing support and resources from the clinic eased barriers preventing access to care after immigrating to the United States. "Here, they help you; here, they send you a taxi; here, they look for you.",

Encountering positive examples of people living well with HIV can help overcome fear, combat stigma, and improve one's mood. One participant recalled, "What really did help me a lot was when I spoke with [a peer with HIV] because he was in my same situation and I saw him in such good spirits. I said to myself, 'He doesn't look sick [laughter]. He's moving a thousand miles an hour!' I'd say, 'No. I can do it, too,' and it helped me a lot to talk to him." The connection among peers with HIV was identified as a unique 
relationship, separate and distinct from the relationship between patients and providers. As one participant stated, "Sometimes, with the title of doctor or social worker, psychologist-I have always thought that they're barriers. Sometimes the titles put a person living with HIV in a different rank... There's a connection; I can identify more and want to talk more with a person who is HIV-positive because I think that I will feel more at ease than I will with a provider."

\section{Discussion}

Among this population of Latinx PLWH in care, themes of language barriers, cultural barriers, inadequate interpreter services, intersectional stigma, and isolation were common (Fig. 1). Many of these challenges to care have previously been seen in studies of Latinx PLWH, ${ }^{10,13-15,23-25}$ although the pervasive sense of isolation and lack of community may be more pronounced in our nonurban population with a low density of LEP patients. The current system of care for Spanish-speaking Latinx PLWH is not meeting this population's needs. Systematic changes are needed to provide equitable care to all PLWH (Table 2).

Language was the most commonly cited barrier. Language discordance between providers and patients results in poorer quality of care, ${ }^{26}$ and Latinx patients may attempt to "get by" with inadequate English for fear of discrimination or being a burden. ${ }^{27}$ The ideal solution is language concordance between providers and patients. ${ }^{28,29}$ However, bilingual providers may not be readily available for low-density minority language populations. Increasing the bilingual provider workforce is a worthy goal, ${ }^{25}$ but this change cannot be enacted immediately. Additionally, language impacts all stages of care, not only the patient/provider interaction.

Ensuring equitable access to care requires that language services are available at every interaction, from telephone encounters, through registration, to delivery of follow-up instructions. In addition, all staff providing language services must be properly credentialed. For patients who are motivated and interested in learning English, active referrals to

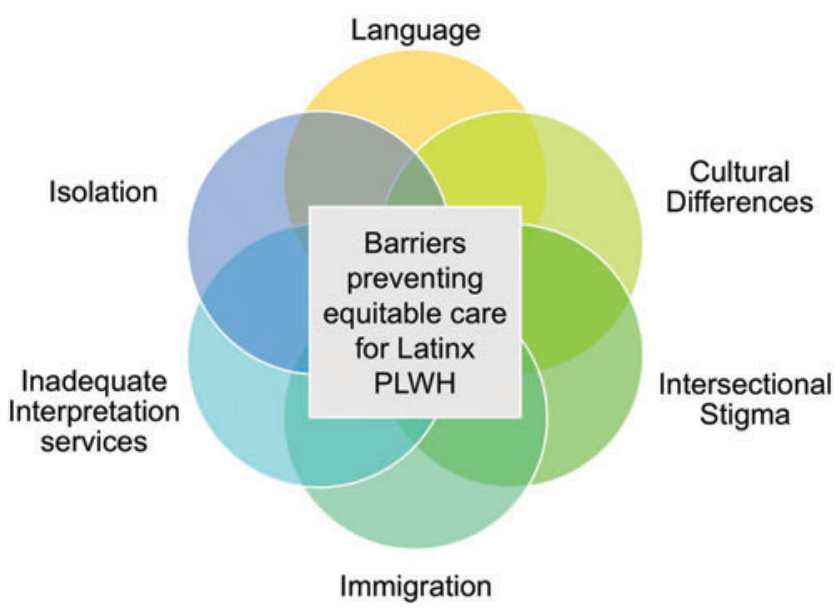

FIG. 1. Intersectional barriers for Latinx PLWH in nonurban South. PLWH, people living with HIV. Color images are available online. language classes should be provided, as some patients identified learning English to be an empowering experience.

In this population of primarily foreign-born Latinxs, cultural differences in the expression of medical concerns and illness impacted patient/provider relationships. While closely tied to language, low quality care among LEP patients also stems from cultural differences. ${ }^{30}$ Literature on HIV prevention among Latinxs describes how cultural norms related to sexuality, gender roles, and social reluctance may limit discussion related to sexual identity and sexual activity. ${ }^{25}$ Diversifying care teams to include members of minority language populations may provide a broader range of perspectives. It is also important for providers to recognize the diversity within minority language populations, given the heterogeneity of cultures among Spanish-speaking individuals.

Interpreter services are often suggested as the solution to address language and cultural barriers among LEP patients. While access to interpreter services is mandated under law $^{31}$ for the $8.7 \%$ of the U.S. population who are LEP, ${ }^{32}$ reimbursement for language services is variable ${ }^{11}$ and language services remain underutilized. ${ }^{33-38}$ LEP patients who receive care with professional interpreters have higher satisfaction with care, higher quality of care, and attend more visits than those using untrained interpreters, such as family members. ${ }^{11,39}$ Yet, interpreters alone cannot overcome language barriers, ${ }^{23}$ as seen in the underutilization and inadequate interpretation services experienced by our participants. This is despite our clinic being located in a large tertiary care health system where interpreters are available at all times. Unanticipated challenges included patient concerns regarding confidentiality and insensitive behavior by interpreters. HIV, a disease associated with a high degree of stigma, may be more impacted by concerns regarding confidentiality and privacy than other medical conditions. The resulting mistrust in interpreter services can impact engagement with care. ${ }^{24}$

We suggest structural changes to the provision of interpreter services for PLWH. First, we recommend that the RWHAP develop specialized training for interpreters who provide services for a highly stigmatized population to ensure accurate interpretation and interpreter familiarity discussing HIV and other sexually transmitted infections.

Specialized training may help address the intersectional stigma these patients face. RWHAPs provide interdisciplinary care, and national guidelines regarding care of Latinx patients recommend including interpreters as members of the care team. ${ }^{30,40}$ The presence of specially trained interpreters embedded in the clinic would meet these recommendations. Second, care teams should acknowledge that patients will be differentially affected by the presence of an interpreter in the room, and particularly in low-density nonurban populations, interpreters may be known to patients. For certain patients, alternative methods of interpretation, including telephone interpreters, or interpretation through certified language technology programs, such as video interpretation, may be preferred to in-person interpreters. There should be a specially trained pool of interpreters available for this option. This could be a centralized resource supported by the RWHAP. A telehealth alternative to access trained interpreters may also be more practical for low-density populations that may not have sufficient patient volume for a dedicated specially trained in-person interpreter. Third, interpreters can serve as cultural brokers, ${ }^{41}$ and training could 
ensure interpreters are well poised to recognize and address the culturally diverse Spanish-speaking population. Additionally, visits requiring interpretation services require more time. ${ }^{42}$ Clinics should create a policy that allows more time for visits with LEP patients, as this may help mitigate barriers. Finally, to ensure improvement in the use of language services, the appropriate utilization of interpretation services should be evaluated, tracked, and reported as a quality metric for patient care and safety within the clinic. ${ }^{30,31}$

Relationships were the primary source of motivation to overcome isolation and barriers. In the Latinx population, "familismo," a cultural value that emphasizes close family connections and loyalty, is a central cultural concept. The sense of isolation felt by many Latinx PLWH may be particularly pronounced given the cultural importance of familismo. ${ }^{25}$ Social support and disclosure of status to members of one's social network improves retention in care. ${ }^{43}$ "Confianza," or trusting relationships, is a cultural theme in care, as supported by our participants who sought a sense of warmth with providers. ${ }^{44}$ Case management can be an effective means to build social support in Latinx PLWH. ${ }^{45}$

For our participants, positive examples of people living well with HIV were transformative in their lives. Opportunities to interact with peers living with HIV and to build community can be limited in low-density minority language populations. Mobile health interventions, such as virtual support groups or smart phone applications allowing for interaction among Spanish-speaking PLWH as well as between PLWH and care providers, could provide an opportunity to build a virtual community of peer support. ${ }^{46}$ Virtual support groups that include peers living well with HIV can also provide examples of positive self-image to help others who may be struggling with internalized stigma. ${ }^{47}$

Finally, while directly mentioned by only a handful of patients to be a barrier, immigration underpins many of the emerging themes. Immigration is one part of the intersectional stigma faced by this population. Stigma, a high concern among Spanish-speaking Latinx PLWH, ${ }^{48}$ results in worse outcomes for PLWH. ${ }^{49}$ Before 2010, HIV infection could be a reason to bar entry into the United States, ${ }^{25}$ a fact known by at least two participants. Under the public charge rule, immigrants who utilize some public programs, or those with certain illnesses commonly impacting PLWH, may be deemed ineligible for entry into the United States or adjustment to legal permanent resident status. ${ }^{50,51}$ Particularly for undocumented immigrants, fear of deportation impacts social determinants of health. Undocumented immigrants face food insecurity, limited access to health care, unstable housing, difficulty finding employment, and mistrust available services. $^{25,52}$ Following expansion of the Affordable Care Act (ACA), the national uninsured population decreased. However, undocumented immigrants were excluded from ACA, ${ }^{53}$ and Latinxs are the racial/ethnic group with the highest uninsured rate at $17 \% .{ }^{54}$ Recent changes to the public charge policy are predicted to result in decreased insurance coverage among immigrant families. ${ }^{55}$ Provision of immigration legal services has been proposed as a structural intervention for HIV care. ${ }^{52}$

Our study has few key limitations. Our participants are all born outside the continental United States and recruited from two sites. Therefore, the results may not be generalizable to native-born Latinx PLWH or a broader region. Some par- ticipants spoke English as well as Spanish, which may influence their perceptions of care particularly as related to language and interpreter services.

\section{Conclusions}

To end the HIV epidemic in the United States and reach 90-90-90, we must improve care for populations disproportionately affected by the HIV epidemic, including Latinx PLWH and PLWH in the South. Health care systems must acknowledge the mistrust and perceptions of discrimination that Latinx patients feel in health care settings, diversify the workforce, and increase access to culturally and linguistically competent care ${ }^{25}$ to create a culture where the equitable care of minority language populations is expected. ${ }^{56}$ Developing specialized training in HIV and sexually transmitted infections for interpreters in RWHAP HIV clinics and creating a centralized pool of specially trained interpreters available for telehealth interpretation may be valuable investments to achieve these goals and help end the HIV epidemic.

\section{Author Contributions}

J.E.S., K.A.M., T.F., and R.D. conceived of the study. K.P.G. and D.A.V. performed the interviews. K.P.G., S.K., T.F., and J.E.S. coded and analyzed the data. R.D. supervised. J.E.S. wrote the initial article, which was revised and reviewed by all authors. All authors approve of the final version.

\section{Acknowledgments}

The authors thank the University of Virginia Ryan White HIV Clinic patients. They also thank the University of Virginia Ryan White HIV Clinic staff, the PositiveLinks team, and Nova Salud. Special thanks to Ava Lena Waldman for assistance with developing the study protocol and Eeshani Thomas for her work in data coding.

\section{Author Disclosure Statement}

K.A.M. reports stock ownership in Gilead Sciences, Inc., R.D. provides consulting services to Warm Health Technologies, Inc., on activities unrelated to this work. K.A.M., R.D., and J.E.S. have received funding from Gilead Sciences, Inc., for an investigator-initiated trial unrelated to this work. The other authors report no disclosures.

\section{Funding Information}

This work was supported by the National Institute of Allergy and Infectious Diseases at the National Institutes of Health (grant number T32 AI007046-41 to J.E.S., grant number K08AI136644 to K.A.M.). The Virginia Department of Health (VDH) also provided funding through Ryan White Part B special projects funding. The content is solely the responsibility of the authors and does not necessarily represent the official views of the NIH or of VDH.

\section{Supplementary Material}

Supplementary Table S1 Supplementary Table S2 


\section{References}

1. US Hispanic population reached new high in 2018 | Pew Research Center. Available at https://www.pewresearch.org/ fact-tank/2019/07/08/u-s-hispanic-population-reached-newhigh-in-2018-but-growth-has-slowed/, accessed February 20, 2020.

2. U.S. Department of Health \& Human Services: U.S. Statistics | HIV.gov. Available at https://www.hiv.gov/hiv-basics/ overview/data-and-trends/statistics, accessed March 4, 2020.

3. Dennis AM, Wheeler JB, Valera E, et al.: HIV risk behaviors and sociodemographic features of HIV-infected Latinos residing in a new Latino settlement area in the Southeastern United States. AIDS Care 2013;25:1298 1307.

4. Trepka MJ, Fennie KP, Sheehan DM, Lutfi K, Maddox L, Lieb S: Late HIV diagnosis: Differences by rural/urban residence, Florida, 2007-2011. AIDS Patient Care STDS 2014;28:188-197.

5. El-Sadr WM, Gamble TR, Cohen MS: Linkage from HIV testing to care: A positive test often leads nowhere. Sex Transm Dis 2013;40:26-27.

6. Centers for Disease Control National Center for HIV/AIDS Viral Hepatitis STD and TB Prevention: Latinos | Race/ Ethnicity | HIV by Group | HIV/AIDS | CDC. Available at https://www.cdc.gov/hiv/group/racialethnic/hispaniclatinos/ index.html, accessed March 5, 2020.

7. Office of National AIDS Policy: National HIV/AIDS Strategy for the United States: Updated to 2020. Available at https://files.hiv.gov/s3fs-public/nhas-update.pdf, Published 2015, accessed February 17, 2020.

8. Clark H, Surendera Babu A, Harris S, Hardnett F: HIVrelated mortality among adults ( $\geq 18$ years) of various Hispanic or Latino subgroups-United States, 2006-2010. J Racial Ethn Health Disparities 2015;2:53-61.

9. Gray KM, Valverde EE, Tang T, Siddiqi AeA, Irene Hall $\mathrm{H}$ : Diagnoses and prevalence of HIV infection among Hispanics or Latinos-United States, 2008-2013. Morb Mortal Wkly Rep 2015;64:1097-1103.

10. Martinez O: HIV-related stigma as a determinant of health among sexual and gender minority Latinxs. HIV Spec 2019;11:14-17.

11. Flores G: the impact of medical interpreter services on the quality of health care: A systematic review. Med Care Res Rev 2005;62:255-299.

12. Freeman R, Gwadz MV, Silverman E, et al.: Critical race theory as a tool for understanding poor engagement along the HIV care continuum among African American/Black and Hispanic persons living with HIV in the United States: A qualitative exploration. Int J Equity Health 2017;16:54.

13. Morales-Aleman MM, Sutton MY: Hispanics/Latinos and the HIV continuum of care in the Southern USA: A qualitative review of the literature, 2002-2013. AIDS Care 2014;26:1592-1604.

14. Dang BN, Giordano TP, Kim JH: Sociocultural and structural barriers to care among undocumented Latino immigrants with HIV infection. J Immigr Minor Health 2012;14: 124-131.

15. Garland JM, Andrade AS, Page KR: Unique aspects of the care of HIV-positive Latino patients living in the United States. Curr HIV/AIDS Rep 2010;7:107-116.

16. Hess K, Johnson S, Hu X, et al.: Diagnoses of HIV infection in the United States and dependent areas, 2017. 2017. Available at http://www.cdc.gov/hiv/library/reports/ hiv-surveillance.html, Published November 2018. Accessed February 20, 2020.

17. Centers for Disease Control: HIV in the Southern United States. 2019. Available at http://www.cdc.gov/, accessed February 28, 2020.

18. Centers for Disease Control National Center for HIV/AIDS Viral Hepatitis STD and TB Prevention: HIV surveillance in urban and nonurban areas through 2017. 2017. Available at https://www.cdc.gov/hiv/pdf/library/slidesets/cdc-hivurban-nonurban-2017.pdf, accessed February 20, 2020.

19. Sheehan DM, Trepka MJ, Fennie KP, et al.: Individual and neighborhood predictors of mortality among HIV-positive Latinos with history of injection drug use, Florida, 20002011. Drug Alcohol Depend 2015;154:243-250.

20. Schafer KR, Albrecht $\mathrm{H}$, Dillingham $\mathrm{R}$, et al.: The continuum of HIV care in rural communities in the United States and Canada: What is known and future research directions HHS public access. J Acquir Immune Defic Syndr 2017;75:35-44.

21. Adler NE, Stewart J: Health disparities across the lifespan: Meaning, methods, and mechanisms. Ann N Y Acad Sci 2010;1186:5-23.

22. Pellowski JA, Kalichman SC, Matthews KA, Adler N: A pandemic of the poor: Social disadvantage and the U.S. HIV epidemic. Am Psychol 2013;68:197-209.

23. Murphy DA, Roberts KJ, Hoffman D, Molina A, Lu MC: Barriers and successful strategies to antiretroviral adherence among HIV-infected monolingual Spanish-speaking patients. AIDS Care 2003;15:217-230.

24. Levison JH, Bogart LM, Khan IF, et al.: "Where it falls apart": Barriers to retention in HIV care in Latino immigrants and migrants. AIDS Patient Care STDS 2017; 31:394-405.

25. Levison JH, Levinson JK, Alegría M: A critical review and commentary on the challenges in engaging HIV-infected Latinos in the continuum of HIV care. AIDS Behav 2018; 22:2500-2512.

26. Ortega AN, Rodriguez HP, Vargas Bustamante A: Policy dilemmas in Latino health care and implementation of the Affordable Care Act. Annu Rev Public Health 2015;36: 525-544.

27. Steinberg EM, Valenzuela-Araujo D, Zickafoose JS, Kieffer E, Decamp LR: The "battle" of managing language barriers in health Care. Clin Pediatr (Phila) 2016;55: 1318-1327.

28. Fernandez A, Schillinger D, Warton EM, et al.: Language barriers, physician-patient language concordance, and glycemic control among insured Latinos with diabetes: The diabetes study of Northern California (DISTANCE). J Gen Intern Med 2011;26:170-176.

29. Ngo-Metzger Q, Sorkin DH, Phillips RS, et al.: Providing high-quality care for limited English proficient patients: The importance of language concordance and interpreter use. J Gen Intern Med 2007;22(SUPPL. 2):324-330.

30. Betancourt JR, Renfrew MR, Green AR, Lopez L, Wasserman M, Associates A: Improving patient safety systems for patients with limited English proficiency: A guide for hospitals. 2012. Available at www.ahrq.gov, accessed January 10, 2020.

31. Guidance for federal financial assistance recipients-Title VI | HHS.gov. Available at https://www.hhs.gov/civilrights/for-individuals/special-topics/limited-english-profici ency/guidance-federal-financial-assistance-recipients-titlevi/index.html, accessed March 4, 2020. 
32. Ryan C: Language use in the United States: 2011. 2013. Available at www.census.gov/acs/www, accessed January 10, 2020.

33. Ramirez D, Engel KG, Tang TS: Language interpreter utilization in the emergency department setting: A clinical review. J Health Care Poor Underserved 2008;19:352-362.

34. Benda NC, Fairbanks RJ, Higginbotham DJ, Lin L, Bisantz AM: Observational study to understand interpreter service use in emergency medicine: Why the key may lie outside of the initial provider assessment. Emerg Med J 2019;36: $582-588$.

35. Diamond LC, Schenker Y, Curry L, Bradley EH, Fernandez A: Getting by: Underuse of interpreters by resident physicians. J Gen Intern Med 2009;24:256-262.

36. DeCamp LR, Kuo DZ, Flores G, O’Connor K, Minkovitz CS: Changes in language services use by US pediatricians. Pediatrics 2013;132:e396-e406.

37. Vela MB, Fritz C, Press VG, Girotti J: Medical students' experiences and perspectives on interpreting for LEP patients at two US medical schools. J Racial Ethn Health Disparities 2015;3:245-249.

38. Brooks K, Stifani B, Batlle HR, Nunez MA, Erlich M, Diaz $\mathrm{J}$ : Patient perspectives on the need for and barriers to professional medical interpretation. R I Med J (2013) 2016; 99:30-33.

39. Karliner LS, Jacobs EA, Chen AH, Mutha S: Do Professional interpreters improve clinical care for patients with limited English proficiency? A systematic review of the literature. Health Serv Res 2007;42:727-754.

40. Strategies for engaging and retaining Latinos in HIV Care. Available at https://www.mathematica.org/our-publica tions-and-findings/publications/strategies-for-engaging-andretaining-latinos-in-hiv-care, accessed March 4, 2020.

41. Sleptsova M, Hofer G, Morina N, Langewitz W: The role of the health care interpreter in a clinical setting-A narrative review. J Community Health Nurs 2014;31:167-184.

42. Kravitz RL, Helms LJ, Azari R, Antonius D, Melnikow J: Comparing the use of physician time and health care resources among patients speaking English, Spanish, and Russian. Med Care 2000;38:728-738.

43. Wohl AR, Galvan FH, Myers HF, et al.: Do social support, stress, disclosure and stigma influence retention in HIV care for Latino and African American men who have sex with men and women? AIDS Behav 2011;15:1098-1110.

44. Flores G: Culture and the patient-physician relationship: Achieving cultural competency in health care. J Pediatr 2000;136:14-23.

45. Gardner LI, Marks G, Craw J, et al.: Demographic, psychological, and behavioral modifiers of the antiretroviral treatment access study (ARTAS) intervention. AIDS Patient Care STDS 2009;23:735-742.

46. Dillingham R, Ingersoll K, Flickinger TE, et al.: PositiveLinks: A mobile health intervention for retention in HIV care and clinical outcomes with 12-month follow-up. AIDS Patient Care STDS 2018;32:241-250.

47. Flickinger TE, DeBolt C, Xie A, et al.: Addressing stigma through a virtual community for people living with HIV: A mixed methods study of the PositiveLinks mobile health intervention. AIDS Behav 2018;22:3395-3406.

48. Rajabiun S, Rumptz MH, Felizzola J, et al.: The impact of acculturation on Latinos' perceived barriers to HIV primary care. Ethn Dis 2008;18:403-408.

49. Katz IT, Ryu AE, Onuegbu AG, et al.: Impact of HIVrelated stigma on treatment adherence: Systematic review and meta-synthesis. J Int AIDS Soc 2013;16(3 Suppl 2): 18640.

50. San Francisco AIDS Foundation: Final public charge rule threatens health. Available at https://www.sfaf.org/collec tions/breaking-news/public-charge-rule-2019-08-14/, accessed April 7, 2020.

51. The Henry J. Kaiser Family Foundation: Changes to "public charge" inadmissibility rule: Implications for health and health coverage. Available at https://www.kff .org/disparities-policy/fact-sheet/public-charge-policies-forimmigrants-implications-for-health-coverage/, accessed April 7, 2020.

52. Yamanis TJ, Zea MC, Ramé Montiel AK, et al: Immigration legal services as a structural HIV intervention for Latinx sexual and gender minorities. J Immigr Minor Health 2019;21:1365-1372.

53. del Rio C: Latinos and HIV care in the Southeastern United States: New challenges complicating longstanding problems. Clin Infect Dis 2011;53:488-489.

54. Cohen RA, Martinez ME, Zammitti EP: Health insurance coverage: Early release of estimates from the National Health Interview Survey, January-March 2017. Available at https://www.cdc.gov/nchs/nhis.htm, accessed February 20, 2020.

55. The Henry J. Kaiser Family Foundation: Health coverage of immigrants. Available at https://www.kff.org/disparitiespolicy/fact-sheet/health-coverage-of-immigrants/, accessed April 7, 2020.

56. Green AR, Nze C: Language-based inequity in health care: Who is the "poor historian"? AMA J Ethics 2017;19:263271.

Address correspondence to: Jacqueline E. Sherbuk Division of Infectious Diseases and International Health University of Virginia PO Box 801379 Charlottesville, VA 22908 USA

E-mail: jes2nk@virginia.edu 